\title{
Single cervical spinal extramedullary tuberculous lesion in a young female - case report
}

\author{
Alexandra Bibirita ${ }^{1}$, Alexandru Nicolae', Adrian Dumitru², Daniel Mihai Teleanu'1,3 \\ ${ }^{1}$ Neurosurgery I Clinic, Emergency University Hospital, Bucharest, Romania \\ ${ }^{2}$ Department of Pathology, Emergency University Hospital, Bucharest, Romania \\ ${ }^{3}$ Department of Neurosurgery, "Carol Davila" University of Medicine and Pharmacy, Bucharest, \\ Romania
}

\begin{abstract}
Skeletal tuberculosis is the main extrapulmonary site for Mycobacterium tuberculosis infection disemination. As for the nervous sistem tuberculosis or spinal tuberculous disease in particular, the osseos elements are more frequently involved than the cerebral tissue or the spinal cord. We present the case of a previously healthy 32 year old female with no known TB history, reffered to our clinic for neck pain thought to be due to degenerative spinal disc disease. In the absence of general signs of the disease, the right surgical management and biopsy were the key factors for corect diagnosis.
\end{abstract}

Keywords: spinal tuberculosis, nervous system tuberculosis, cervical neuralgia, tuberculous granuloma

\section{INTRODUCTION}

Extrapulmonary tuberculosis (TB), especially spinal TB, is a very rare entity nowadays, even in Romania, a country that as of 2020 is still an endemic zone for pulmonary tuberculosis according to the latest ECDC reports [1].

One in five patients infected with Mycobacterium tuberculosis are expected to manifest extrapulmonary tuberculosis at some point in life $[2,3]$. The most common extrapulmonary form of this disease is the skeletal type, with a predilection for the spine, with osseos TB being more common than spinal cord TB.

Nervous system tuberculosis is still a major challenge, both in developed and underdeveloped countries, not only with regard to the surgical management but also when it comes to reaching the right diagnosis [3].
Spinal TB presents with local pain and spinal deformity that leads to gait disfunction, sometimes followed by sensory or motor disfunction in connection with the degree of compression or invasion $[3,4]$.

Skeletal TB is primarly discoved on plain $\mathrm{X}$-rays, but further imagery studies such as MRI (both plain and with intravenous contrast enhancement) help characterise the lesion and clarify its etiology $[5,6]$.

We present the case of a previously healthy 32 year old female that addressed her family physician for cervical pain that was initially managed as degenerative cervical disc disease.

\section{CASE PRESENTATION}

A 32 year old female patient, previously healthy and with no significant medical history presented 
with intermitent neck pain iradiating to the shoulders bilaterally, accompanied by tingling on the same site. Symptoms started suddendly roughly 3 months prior to admission. Her primary physician had started pain mangement with anti-inflammatory drugs and muscle relaxants, without much relief.

Patient complaints were intermitent cervical C3 and $\mathrm{C} 4$ neuralgia and tingling in the neck and shoulders over nearly three months.

On neurological examination the patient did not have neurological deficits, muscle tone was normal, reflexes within normal limits with neither upper nor lower motor neuron signs.

Local examination revealed neither stiff neck nor dystonia.

She underwent a cervical spinal and cerebral MRI, both plain and with intravenous contrast enhancement, that revealed a unique lesion - a space occupying mass located extramedullary, extradural and posteriorly in the cervical spinal canal between $\mathrm{C} 2$ and $\mathrm{C} 4$, about 2 centimeters long and 0.5 $\mathrm{cm}$ thick. Bone structure at this level was within normal limits, but the C2 laminae was thiner, which is thought to be a result of local bone compression. Said lesion was not the contrast capturing type (figure1).
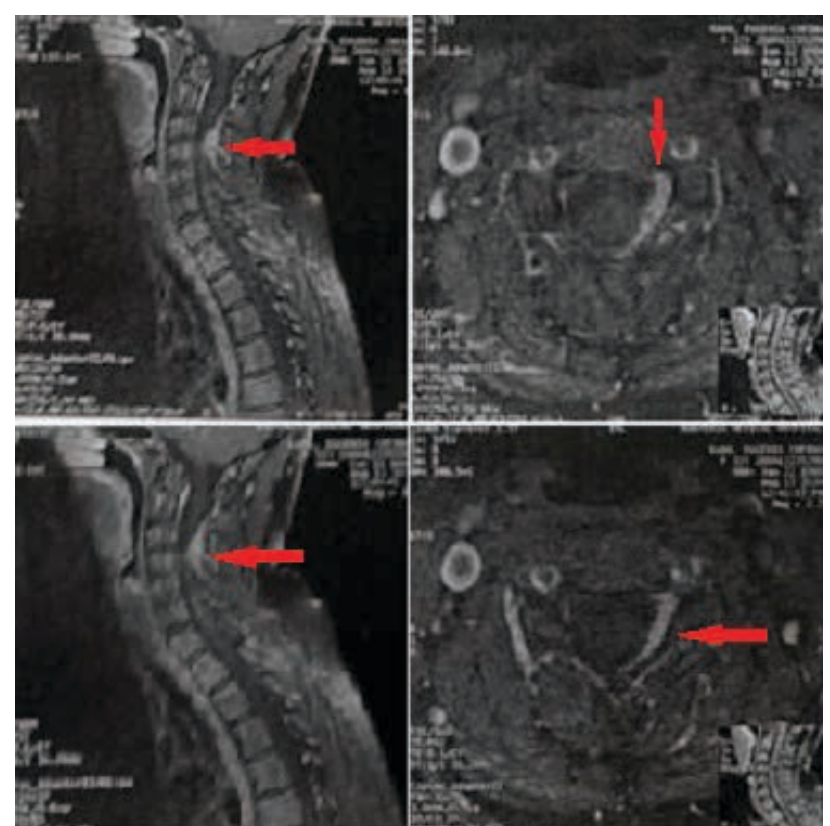

FIGURE 1. MRI sequences of the cervical spine. Arrows point to the extradural C3-C4 mass

Pre-operatory workup, including blood work and pulmonary films did not reveal any abnormal findings: no anemia, no inflamatory markers, no suspicious radiological findings.
The patient underwent surgery and C3-C4 laminectomies were performed in order to expose and access the spinal canal. We immediately discovered the space occupying lession seen on the MRI exam, which was gray-yellow and soft, avascular, about $2.5 \mathrm{~cm}$ long and $0.5 \mathrm{~cm}$ thick, slightly thicker and far more spread to the left side. It quickly allowed for a dissection plane to be found and complete removal was easily possible with complete preservation of the cervical dura mater integrity. The dura mater at this level did not look infiltrated and its colour and aspect were normal (figure 2).

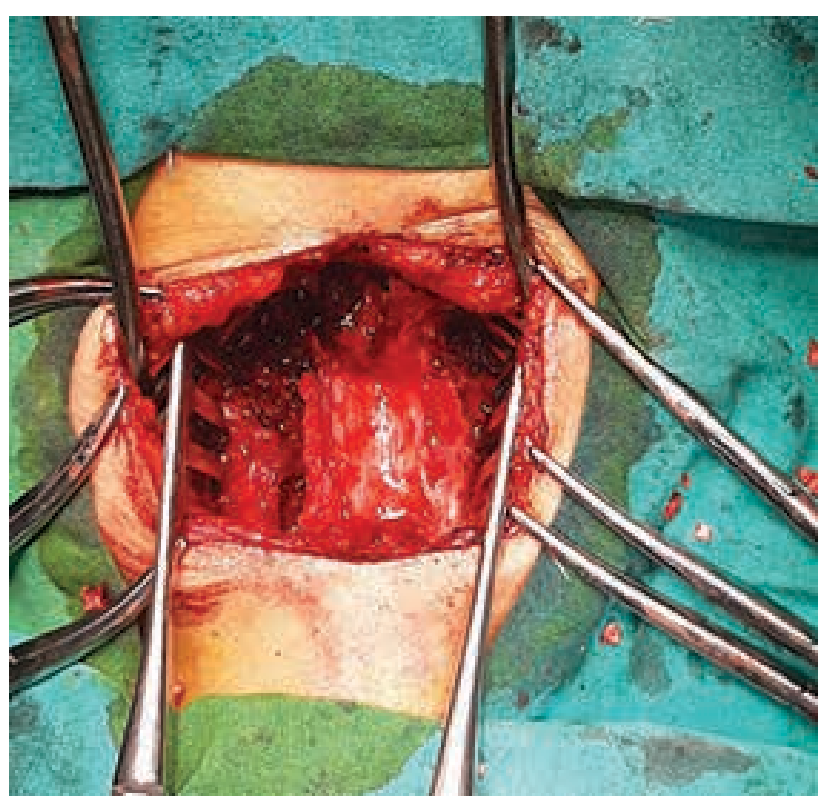

FIGURE 2. Intraoperative aspect of the cervical dura mater - no infiltration

The intraoperative pathology report raised a high suspicion of a tuberculous granuloma (figure 3 and 4) and since no intradural nor intramedullary abnormalies were found on the imagery, the decision was made not to open the dura mater, in order not to spread any germs through CSF fluid to the central nervous sistem.

\section{DISCUSSION}

The surgical management of the space occupying lesion in this patient's cervical spinal canal consisted of neurological decompression and total and safe ablation of the lesion.

Symptomatic lesions in the spinal canal need to benefit from a biopsy, at least, if surgical removal is not possible. Although the patient did not present any sign of systemic disease, spinal malignancy was one of our preoperatory concerns $[6,7]$. 


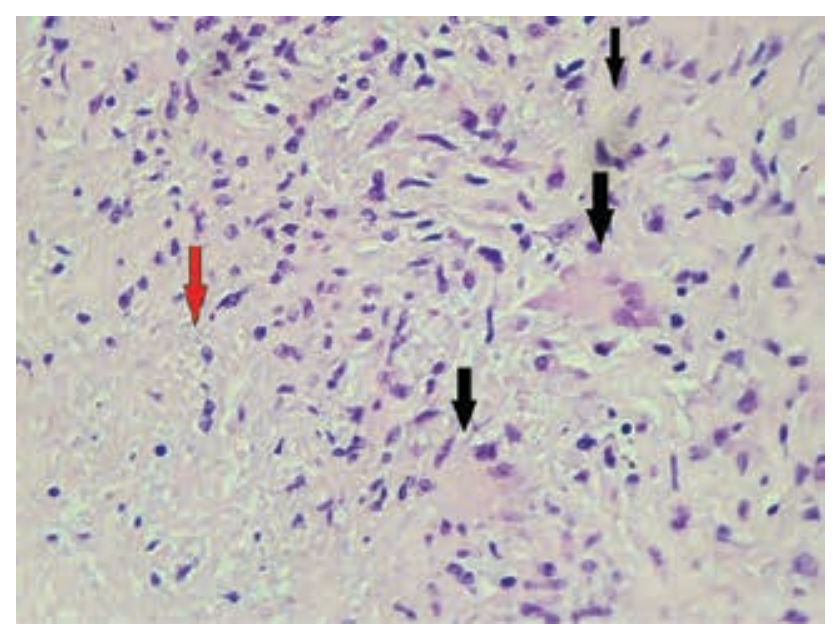

FIGURE 3. Pathology sequence showing tuberculous granuloma. Black arrows point to giant cel with multiple nuclei. Red arrow points to central caseous necrosis

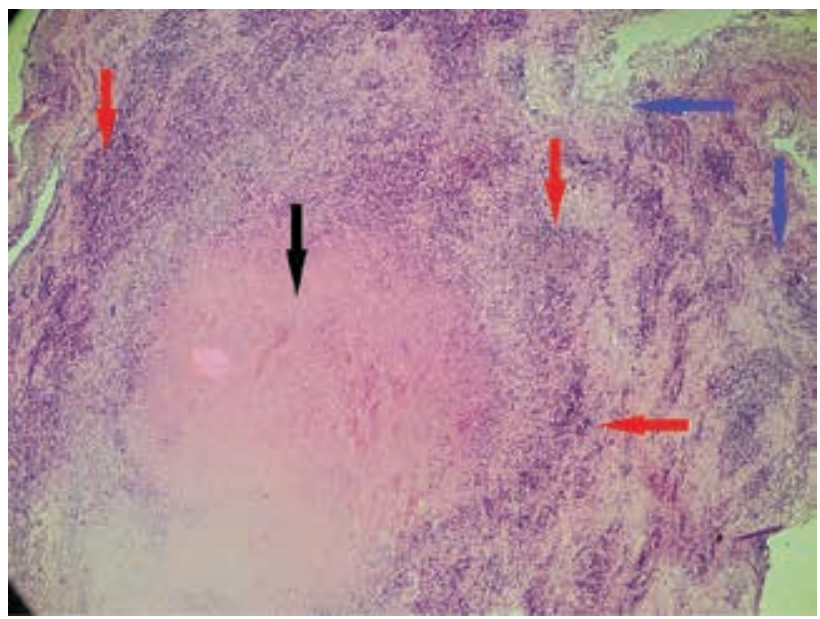

FIGURE 4. The pathology findings from the cervical spinal lesion as seen under microscope - a tuberculous granuloma. Black arrow points to caseous necrosis. Blue arrows point to external ring of cells - e.g. lymphocytes

Following the histopathology definitive report we completed the scanning of the patient's spine

Conflict of interest: none declared

Financial support: none declared

\section{REFERENCES}

1. Teka M, Ghozlen HB, Zaier AY, Hnia MB, Naouar N, Abid F. Cervical spine tuberculosis. Pan Afr Med J. 2020 Sep 2;37:7.

2. Ben Hamida MK, Benmohamed O, Bekkay MA, Habboubi K, Bouhdiba S, Kherfani A, Mestiri M. Tuberculosis of the cervical spine. Tunis Med. 2019 Mar;97(3);512-15.

3. Rajasekaran S, Soundararajan DCR, Shetty AP, Kanna RM. Spinal Tuberculosis: Current Concepts. Global Spine J. 2018 Dec;8(4 Suppl):96S-108S.

4. Salvador GLO, Basso ACN, Barbieri PP, Leitao CA, Teixeira BCA, Neto $A C$. Central nervous system and spinal cord tuberculosis: Revisiting an important disease. Clin Imaging. 2020 Jul 29;69:158-168.

5. Misra UK, Warrier S, Kalita J, Kumar S. MRI findings in Pott's spine and correlating clinical progress with radiological findings. Neuroradiology. 2020 Jul;62(7):825-832.

6. Tan TSE, Mahmood RD. Atypical spinal tuberculosis mimicking malignancy: the utility of multimodality imaging evaluation. Postgrad Med J. 2021 Mar;97(1145):197-198. and brain with MRI imaging [8]. Fortunately no other abnormalities were found.

Commonly, tuberculous lesions in the extramedullary compartment have been described in the last twenty years in patients who had previously suffered from tuberculous meningitis. However, these lesions were intradural and not extradural, as was the lesion we encountered $[9,10]$.

During the postoperative period we completed TB workup with dosing of serum IgM and $\mathrm{IgG}$, with negative results. After surgery the patient no longer had any neurological complaints and was discharged on day three. On discharge the patient was reffered to a pneumology clinic for further treatment.

\section{CONCLUSIONS}

This particular case we encountered stands out mainly because it is about a unique symptomatic lesion in the absence of pulmonary or chronic infectious disease manifestations. The more interesting aspect of the case is that it presented in a young person with no prior disease symptoms, no comorbidities and no immunity disorder. Considering that the patient had not had any prior complaints nor findings that would indicate a TB infection, both primary or disseminated, we conclude that the right imaging studies, followed by the adequate surgical approach and management and a quality pathology report, were our key tools for the right and complete diagnosis. We did not find any lesion of this type and with extramedullary position in more recent literatture.

7. Li Y, Wang Y, Ding H, Zhang N, Ma A, Shi J, Niu N. Pathologic characteristics of spinal tuberculosis: analysis of 181 cases, Int $J$ Clin Exp Pathol. 2020 May 1;13(5):1253-1261.

8. Siddiqui MA, Sartaj S, Rizvi SWA, Khan MJ, Khan IA. Role of Whole-Spine Screening Magnetic Resonance Imaging Using Short Tau Inversion Recovery or Fat-Suppressed T2 Fast Spin Echo Sequences for Detecting Noncontiguous Multiple-Level Spinal Tuberculosis. Asian Spine J. 2018 Aug;12(4):686-690.

9. Sardana R, Sardana NK, Sharma A., Tuberculosis of the axial skeleton mimicking malignancy. J R Coll Physicians Edinb. 2020 Jun;50(2):168-170.

10. Jeong DK, Kwon YM. Intradural Extramedullary Tuberculoma of the Spinal Cord Following Tuberculous Meningitis. Korean J Spine. 2015;12(2):107-110. 